Milena de Barros Viana, Bárbara dos Anjos Rosário, Maria de Fátima Santana de Nazaré, Débora Estadella, Daniel Araki Ribeiro and Glauce Socorro de Barros Viana*

\title{
COVID-19 in age-related neurodegenerative diseases: is there a role for vitamin D3 as a possible therapeutic strategy?
}

https://doi.org/10.1515/revneuro-2020-0074

Received July 15, 2020; accepted September 20, 2020;

published online November 12, 2020

\begin{abstract}
The coronavirus disease (COVID-19), identified in Wuhan, China, on December 2019, was declared a pandemic by the World Health Organization, on March, 2020. Since then, efforts have been gathered to describe its clinical course and to determine preventive measures and treatment strategies. Adults older than 65 years of age are more susceptible to serious clinical symptoms and present higher mortality rates. Angiotensin-converting enzyme 2 (ACE2) is a major receptor for some coronavirus infection, including SARS-COV-2, but is also a crucial determinant in anti-inflammation processes during the renin-angiotensin system (RAS) functioning - converting angiotensin II to angiotensin 1-7. The decline in ACE2 expression that occurs with aging has been associated to the higher morbidity and mortality rates in older adults. These observations highlight the importance of investigating the association between COVID-19 and age-related neurodegenerative disorders, i.e., Parkinson's and Alzheimer's diseases. A possible option to reduce the risk of COVID-19 is vitamin D supplementation, due to its anti-inflammatory and immune-system-modulating effects. It has also been suggested that vitamin D supplementation plays a role in slowing progression of Parkinson and Alzheimer. The present study is a literature review of articles published on the theme COVID-19, Parkinson and Alzheimer's diseases, and the role played by vitamin D. PUBMED, MEDLINE, and EMBASE databases were consulted. Results confirm neurodegenerative and neuroinflammatory effects of COVID-19, aggravated in Parkinson's and Alzheimer's
\end{abstract}

\footnotetext{
*Corresponding author: Glauce Socorro de Barros Viana, Department of Physiology and Pharmacology, Federal University of Ceará (UFC), Fortaleza 60430-275, Ceará, Brazil, E-mail: gbviana@live.com Milena de Barros Viana, Bárbara dos Anjos Rosário, Maria de Fátima Santana de Nazaré, Débora Estadella and Daniel Araki Ribeiro, Department of Biosciences, Federal University of São Paulo (UNIFESP), Santos 11015-020, São Paulo, Brazil
}

patients, and the important role of vitamin D as a possible therapeutic strategy. Nevertheless, randomized controlled trials and large population studies are still warranted.

Keywords: COVID-19; dementia; neurodegeneration; neuroinflammation; vitamin D3.

\section{Introduction}

Coronaviridae (CoVs) are enveloped, single-stranded viruses, which have been shown to cause a series of enteric and respiratory conditions in different animal species, including humans (Fan et al. 2019; Vicenzi et al. 2004). During the last years, CoVs classification has expanded, with the identification and inclusion of new subtypes. Currently, the International Committee of Taxonomy of Viruses (ICTV) classification describes four groups $(\alpha-, \beta$, $\delta$-, and $\gamma$-) and 38 distinct unique species (CTV Virus Taxonomy: 2018 Release).

While $\alpha$ - and $\beta$-CoVs infect mammals, CoVs $\gamma$ - and $\delta$ were shown to be present in different avian species (Morty and Ziebuhr 2020). Since 2002, three major pathogenic subtypes of $\beta$-CoVs have emerged and disseminated worldwide (Fan et al. 2019). SARS-CoV was originated from China, became pandemic in 2002-2003, and infected approximately 8000 individuals with an estimated mortality rate of $10 \%$ ( Ruan et al. 2003). MERS-CoV emerged in the Middle East in 2012 and spread to 27 countries, with an average mortality rate of 35.5\% (WHO 2019).

On December 2019, a new $\beta$-CoV was identified in Wuhan, province of Hubei, China. The new virus, SARS-CoV-2, was recognized as a member of the subgenus Sarbecovirus (family Coronaviridae, genus Betacoronavirus) (Lau et al. 2020). It was also shown to be related to SARS-CoV, which caused the world epidemic in 2003, and to SARS-related-CoVs (SARSr-CoVs), identified in horseshoe bats both in the island of Hong Kong and in mainland China (Ge et al. 2013; Hu et al. 2017; Lau et al. 2005), thus supporting an interspecies transmission. On March 11, 2020, the World Health Organization (WHO) described the new corona virus disease (COVID-19) as pandemic (WHO 2020). 
As demonstrated by recent literature reviews (Harapan et al. 2020; Singhal 2020; Sohrabi et al. 2020; Vieira et al. 2020; Wang et al. 2020), SARS-CoV-2 infection is linked to severe acute respiratory infection and usually causes symptoms of a common cold, such as dry cough, fever, and fatigue. Enteric (i.e. abdominal pain, diarrhea, nausea, vomiting) and neurological symptoms (i.e. headache, dizziness, ataxia) might also accompany the infection. Although most cases are spontaneously resolved, some patients develop a serious clinical condition, including pneumonia, dyspnea, and acute respiratory distress syndrome (ARDS) (with the need for oxygen supplementation) (Harapan et al. 2020; Singhal 2020; Sohrabi et al. 2020; Vieira et al. 2020).

CoVs infection is mediated by the interaction between the viral spike (S) protein, present in the virus envelope membrane, and receptors on target cell surfaces (Hofman and Pöhlmann 2004). The angiotensinconverting enzyme 2 (ACE2) has been recently identified as a major receptor for SARS-CoV-2 (Gheblawi et al. 2020). This enzyme is found in different body tissues, including cardiorespiratory, gastrointestinal, and central nervous system and is a major regulator of the renin-angiotensin system (RAS). It is also a crucial determinant in antiinflammation processes, during the RAS conversion of angiotensin II, the main perpetrator of inflammation, to angiotensin 1-7, which possesses anti-inflammatory properties (Al Gatrif et al. 2020). The expression of ACE2 in cortical neurons and glia makes these cells susceptible to SARS-CoV-2 infection (Baig and Sanders 2020). Thus, there seems to be crucial interactions between ACE2, immunity, peripheral and central inflammation, and cardiovascular disease.

Although all age groups are at risk of contracting COVID-19, older individuals are most susceptible, making aging the most important risk factor for developing COVID-19 (Hou et al. 2019; Naughton et al. 2020). The high risk to the older population to COVID-19 infection can lead to a cytokine storm, characterized by severe systemic elevation of several proinflammatory cytokines (Meftahi et al. 2020) (Figure 1). Older individuals, especially those with hypertension and diabetes, have reduced ACE2 expression and upregulation of the angiotensin II proinflammatory signaling (AlGhatrif et al. 2020; Baig and Sanders 2020; Peña Silva et al. 2012). SARS-CoV-2 infection may accelerate this process, inducing increased secretion of proinflammatory cytokines, which can be actively transported to the central nervous system. After crossing the blood-brain barrier, these cytokines activate glial neuroinflammation responses and increase oxidative stress, damaging cell structures and functions and leading to excitotoxicity (Baig and Sanders 2020).

Neuroinflammation is also known to be a prominent feature of neurodegeneration and plays a major role in neurodegenerative diseases. Immune response and excessive inflammation in COVID-19 may thus accelerate the progression of brain inflammatory neurodegeneration. In fact, previous studies (De Chiara et al. 2012) have shown that viral infection and excitotoxicity play important roles in the genesis of different neurodegenerative conditions, including Parkinson's and Alzheimer's diseases. These observations highlight the importance of investigating the

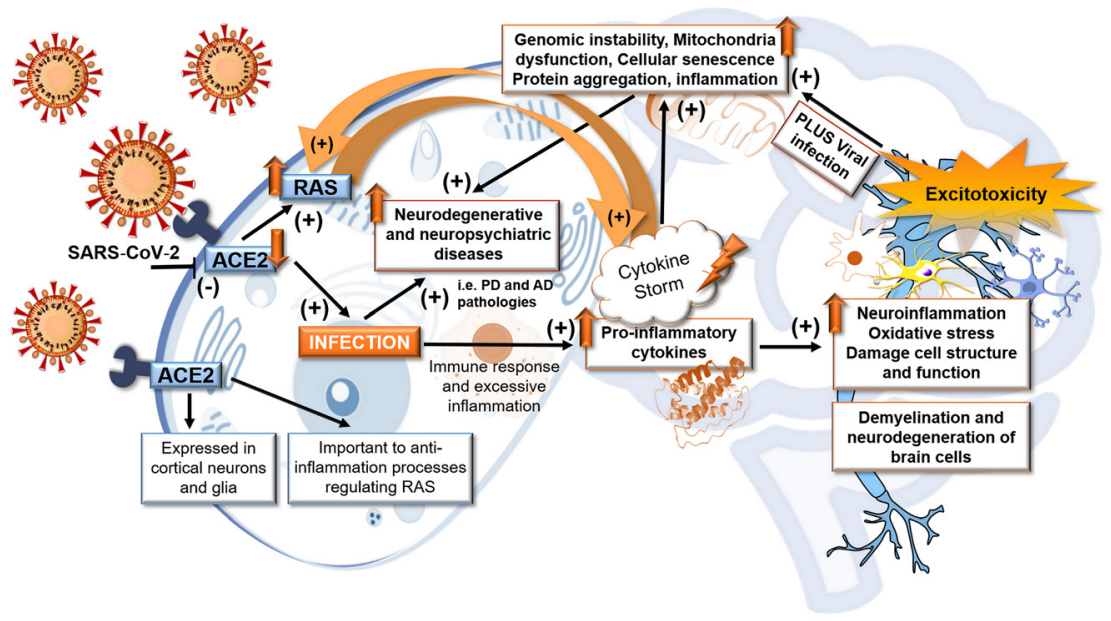

Figure 1: Possible associations between COVID-19 and age-related neurodegenerative diseases.

Reduced ACE2 expression and upregulation of angiotensin II proinflammatory signaling that occur with age, induce a more severe immune response. SARS-CoV-2 infection activates proinflammatory cytokines, which are actively transported to the central nervous system, leading to neuroinflammation and neurodegeneration. Older individuals, especially those with neurodegenerative diseases, are thus more susceptible to the neurological effects of COVID-19.

Abbreviations: RAS (Renin-Angiotensin System); ACE2 (angiotensin-converting enzyme 2); PD (Parkinson's disease); AD (Alzheimer's disease). 
association between COVID-19 and this neurodegenerative disorders.

Apart from that, recent studies (Daneshkhah et al. 2020; Davies et al. 2020; Ebadi and Montano-Loza 2020) have suggested a possible association between low levels of vitamin $\mathrm{D}$, which might be present in the elderly population, smokers, or patients with chronic diseases, and COVID-19 severity and mortality. The active form of vitamin $\mathrm{D}$, calcitriol, has been shown to exhibit significant potent activity against SARS-CoV-2 (Mok et al. 2020). Vitamin D may protect from viral infection and ameliorate the symptoms of COVID-19, including the cytokine storm.

The present review gathered information from clinical randomized trials, reviews, and case reports, regarding the association between COVID-19, neurodegeneration, neuroinflammation, Parkinson and Alzheimer, and vitamin D. The main objective of the study was to summarize available data to allow the development of preventive measures and effective therapeutic interventions.

\section{Materials and methods}

A comprehensive literature search was performed, by two different researchers, in PUBMED, MEDLINE, and EMBASE databases for all kinds of articles. The terms "COVID-19 and neuroinflammation," "COVID-19 and neurodegeneration," “COVID-19 and Alzheimer,” and "COVID-19 and Parkinson” were used. Information was collected from case studies, clinical trials, research studies, and review articles. Letters of correspondence and viewpoints were not considered. Articles that did not deal with the effects of COVID-19 on neuroinflammation, neurodegeneration, and Parkinson or Alzheimer were excluded. Articles that dealt with other viruses from the coronavirus family were also disregarded.

The electronic databases were last searched on June 29, 2020. The main studies are listed in Tables 1-3.

\section{Results}

There were a total of 89 articles. Of those, 66 articles were excluded following the exclusion criteria adopted. Articles that appeared repeatedly in more than one cross-reference were only listed in one of tables. A total of 23 articles were distributed among the three tables.

Table 1 presents studies originated from the crossreference "COVID-19 and neuroinflammation" (5 articles) and "COVID-19 and neurodegeneration" (1 article); Table 2, "COVID-19 and Alzheimer" (4 articles); Table 3, "COVID-19 and Parkinson" (13 articles). Although no articles were found with the cross-reference of the terms "vitamin D" and "COVID-19 and neuroinflammation," "COVID-19 and neurodegeneration," or "COVID-19 and Alzheimer," three articles were found regarding "vitamin D, COVID-19 and Parkinson." Nevertheless, these same articles were already listed in Table 3-Vitamin D, COVID-19, Parkinson.

\section{Discussion}

The main objectives of the present review were to address information derived from case reports, clinical trials, research studies, and review articles on the relationship between 1. COVID-19 and neuroinflammation; 2. COVID-19 and neurodegeneration, 3. COVID-19 and Parkinson's disease, 4. COVID-19 and Alzheimer's disease, and 5. COVID-19 and vitamin D. Results showed that several previously published studies were viewpoints and letters to the Editor, which were excluded. Some were reviews or case reports. Very few were randomized controlled trials. We will then present the main studies that resulted from our cross-reference searches and discuss important information, gathered from the literature on the aforementioned selected topics.

The cross-reference of the terms "COVID-19 and neuroinflammation" resulted in the finding of 12 published studies. From those, only five were included according to the criteria adopted. They were all literature reviews (Herman et al. 2020; Mao et al. 2020; Ogier et al. 2020; Raony et al. 2020; Serrano-Castro et al. 2020). Ogier and coworkers (2020) investigated, on the basis of previously published reports, the relationship between SARS-Cov-2 infection and inflammation of brain parenchyma, leading to progressive brain damage, demyelination, and neurodegeneration of brain cells. The authors suggest that these neurological alterations require a long-term follow-up of patients. Similar concerns were raised by the other reviews (Herman et al. 2020; Mao et al. 2020; Raony et al. 2020; Serrano-Castro et al. 2020). According to Serrano-Castro and coworkers (2020), there is an impact of this infection on the onset and progression of neurodegenerative and neuropsychiatric diseases of neuroinflammatory origin that should be regarded as the potential cause of a delayed pandemic.

Similarly, from a total of 12 articles, only one review article remained (following our exclusion criteria) with the cross-reference of the terms "COVID-19 and neurodegeneration" (Singal et al. 2020). This article was also included in Table 1. Singal and coworkers (2020) investigate virulence, epidemiology, molecular, and neuropathological pathways of the virus. Placental transmission and possible therapeutic strategies are also mentioned. Among 


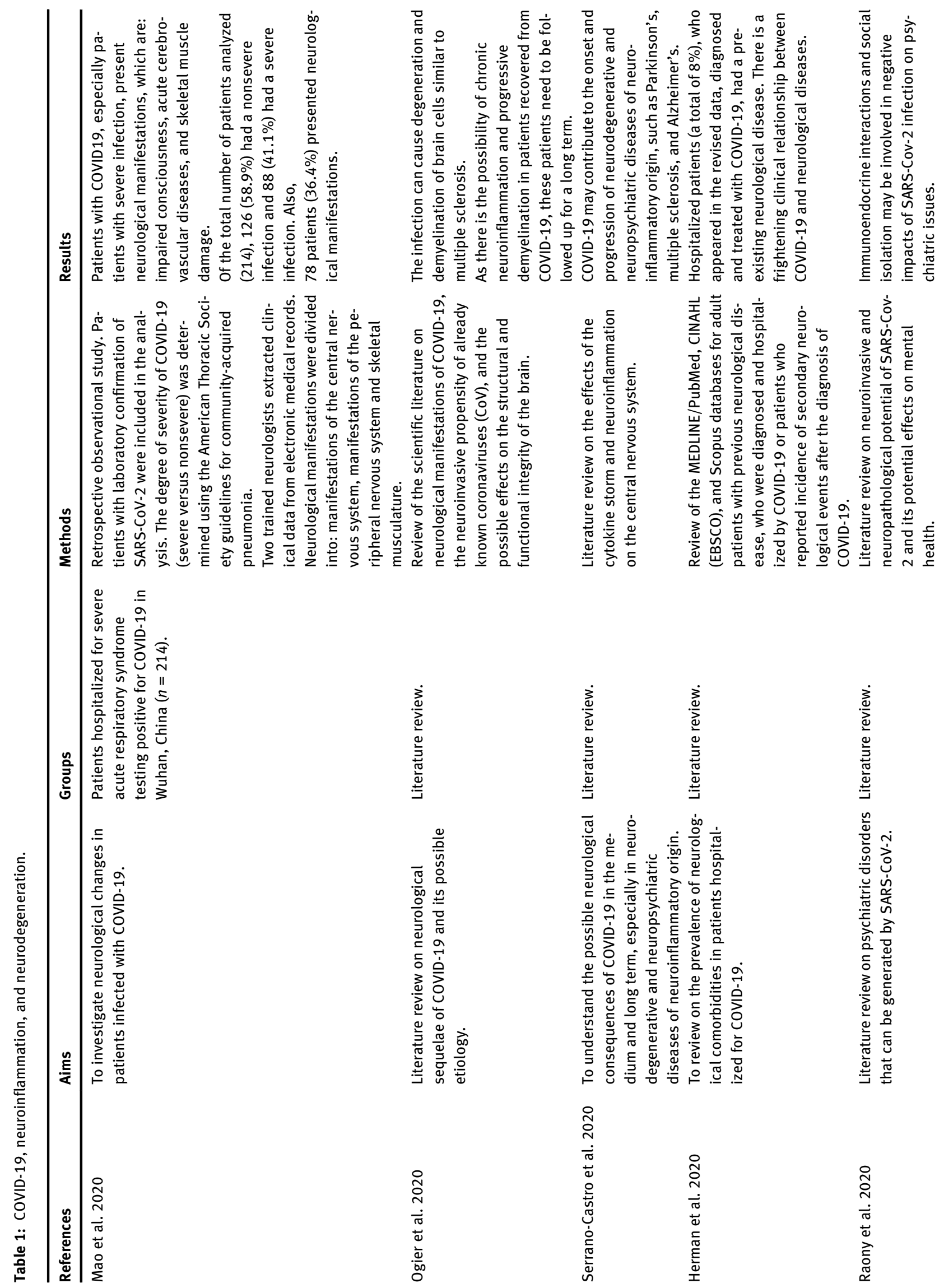




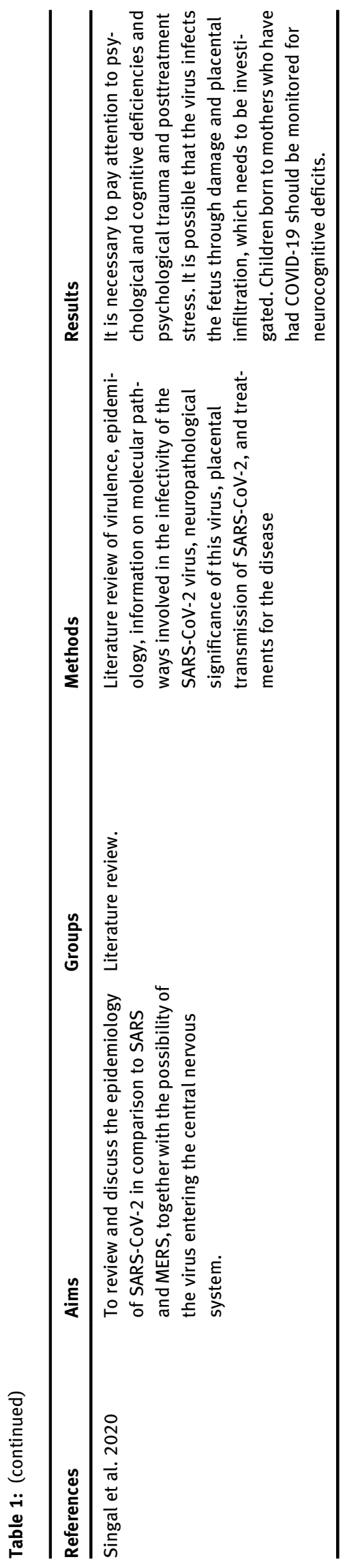

the therapeutic strategies, the authors cite ACE2 inhibitors and angiotensin receptor blockers, antimalarial drugs (such as Chloroquine and Hydroxychloroquine), and antiParkinsonian and anti-Alzheimer drugs, such as adamantanes (amantadine, memantine), to treat the neuropathological symptoms derived from Sars-Cov-2 infection.

With the exclusion of viewpoints and corresponding letters, there were only four studies resulting from the crossing of the terms "COVID-19 and Alzheimer." One of them was a clinical analysis of a day-by-day case of an 83-year-old woman with COVID-19/Alzheimer (Isaia et al. 2020). The main conclusions were: 1 ) the report of atypical symptoms, i.e., diarrhea and drowsiness, and 2) the need for adequate supportive measures to improve survival. Worsening of neuropsychiatric symptoms in COVID-19/ Alzheimer's patients was described also by two clinical reports (Boutoleau-Bretonnière et al. 2020; Lara et al. 2020). Interestingly, an experimental research study (Viel et al. 2020), determining the levels of IL-6 in cultures of human astrocytes, suggests that lithium may constitute a novel potential therapeutic to help COVID-19/Alzheimer's patients, reducing the virulence of SARS-CoV-2.

Regarding "COVID-19 and Parkinson," there was a total of 13 articles listed. From those, six were clinical interviews (Fasano et al. 2020; Prasad et al. 2020; Rejdak and Grieb 2020; Salari et al. 2020; Shalash et al. 2020; Zipprich et al. 2020). These studies emphasize the influence of psychological symptoms on the aggravation of the clinical condition of the patients (Prasad et al. 2020; Salari et al. 2020; Shalash et al. 2020) and the worsening of motor symptoms (Prasad et al. 2020; Zipprich et al. 2020). These were also the general conclusions of three literature reviews (Bhidayasiri et al. 2020; De Felice et al. 2020; Garg and Dhamija 2020), a research study conducted with two deep brain stimulation-treated Parkinson/COVID-19 patients (Hainque and Gabli 2020) and a clinical observational study performed with COVID-19 positive and nonpositive tested Parkinson's patients (Cilia et al. 2020). Possible therapeutic strategies were also cited. Rejdak and Grieb (2020) evaluated, through the use of clinical questionnaires, the effects of adamantanes in patients with COVID-19 and multiple sclerosis, Parkinson, or cognitive impairment and concluded that the drugs indeed possess protective effects in those individuals suffering from neurological disorders. Fasano and coworkers (2020) also used clinical interviews to identify whether patients with Parkinson's disease were at higher risk for COVID-19, what were the risk factors and clinical manifestations. They concluded that although mortality rates did not differ from the general population, neurological symptoms require attention. According to the authors, a possible important 


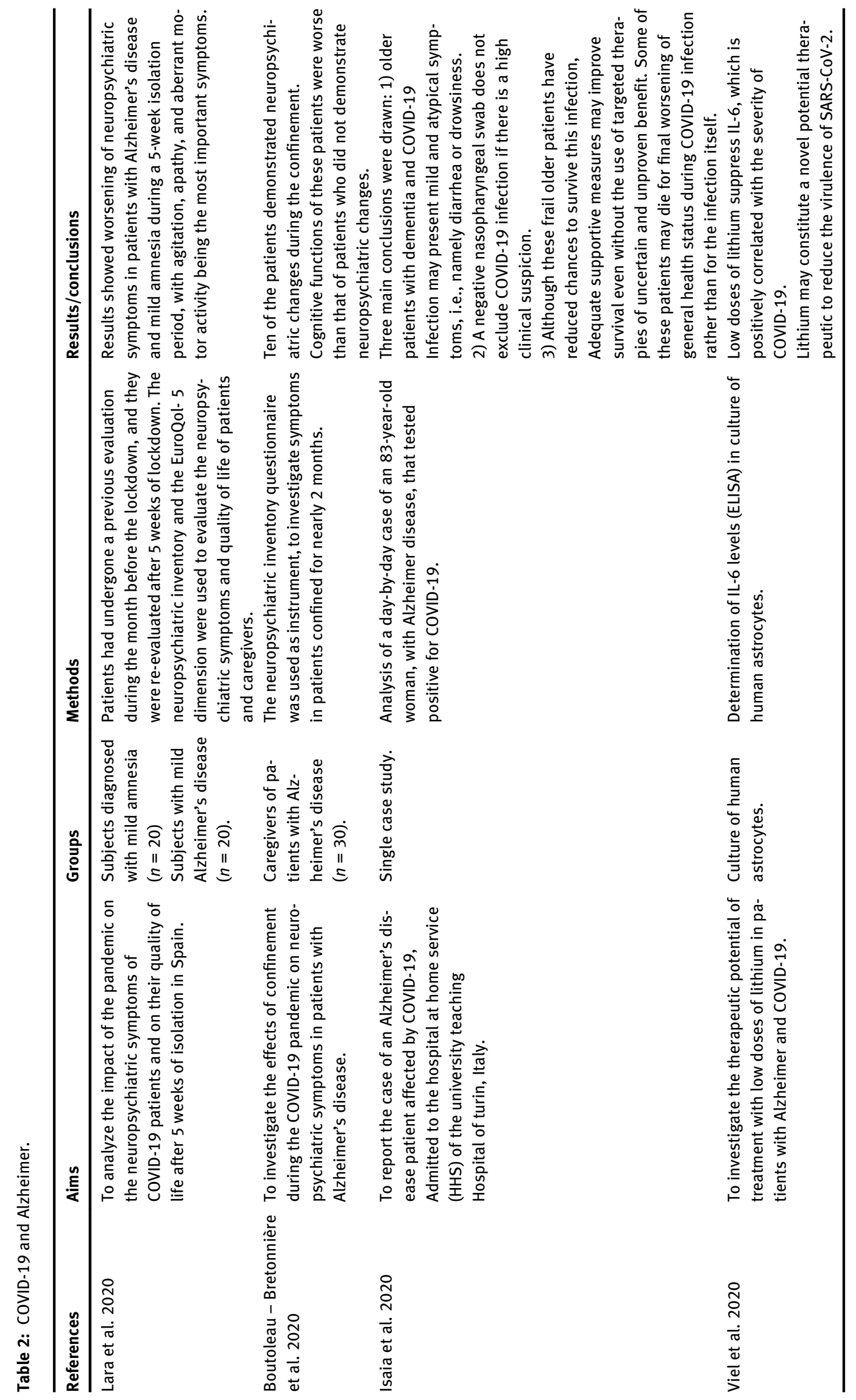




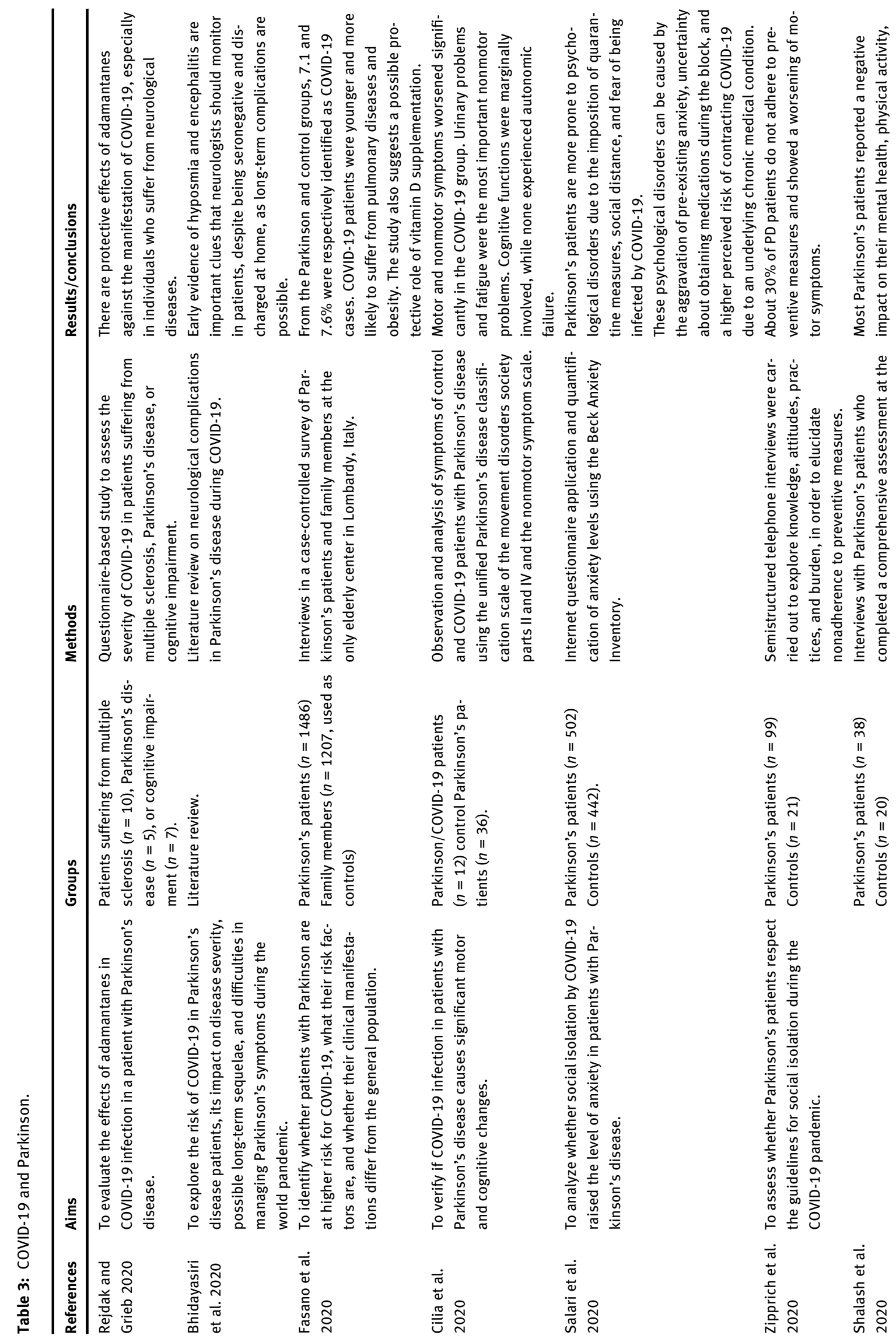




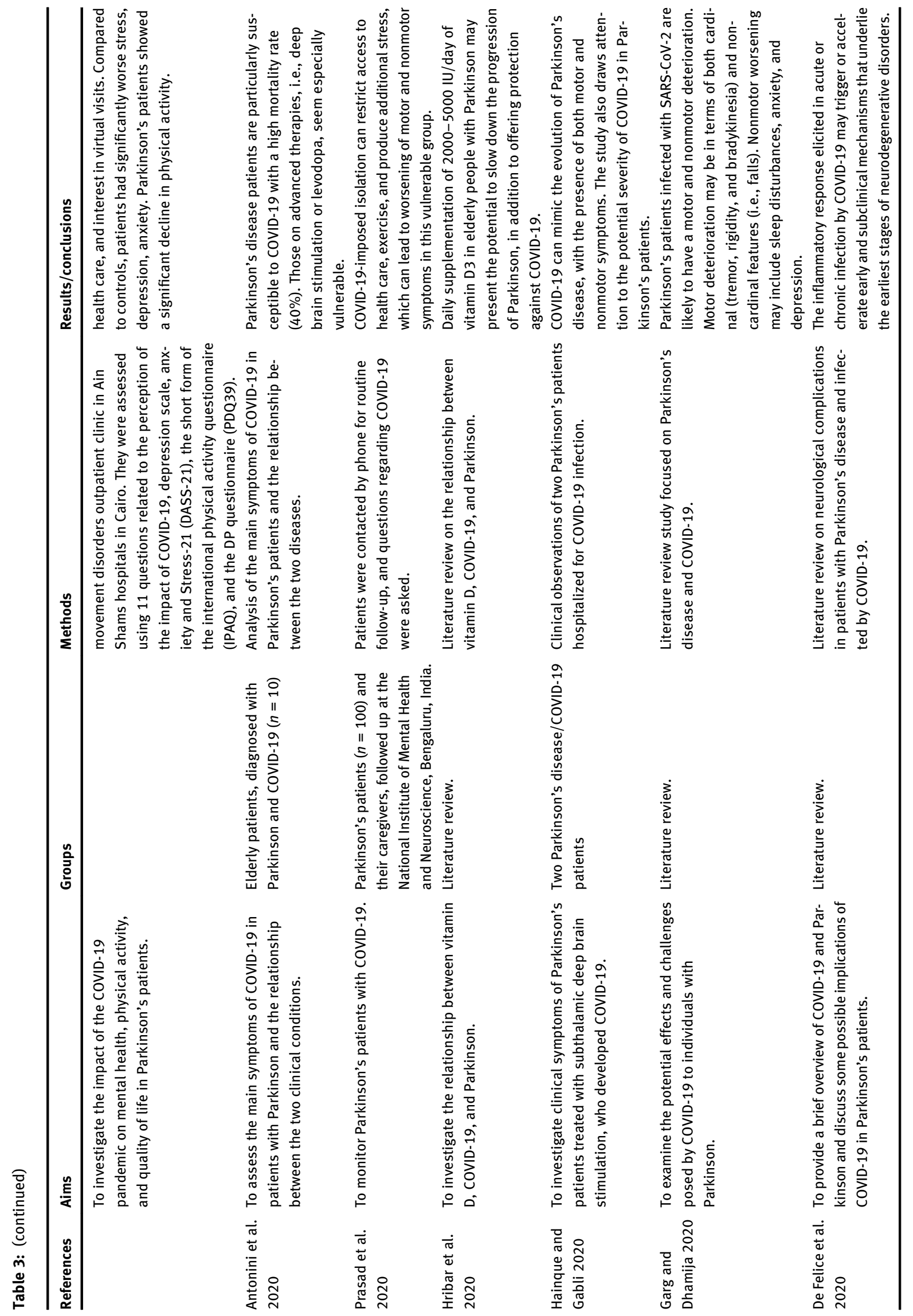


role for vitamin D supplementation warrants future studies. This is also the conclusion of a literature review conducted by Hribar and coworkers (2020).

With that in mind, we cross-referenced the terms vitamin D, COVID-19 and neuroinflammation, neurodegeneration, Alzheimer or Parkinson. These, however, were the only two reports found (Fasano et al. 2020; Hribar et al. 2020). Nonetheless, some important considerations will be drawn in the next paragraphs regarding the possible therapeutic role played by vitamin D in COVID-19 neurological symptoms.

Vitamin D was firstly characterized as a vitamin in the 20th century, and now it is recognized as a prohormone. The two major forms of vitamin $\mathrm{D}$ are vitamin $\mathrm{D}_{2}$ (ergocalciferol, derived from plant sources) and vitamin $\mathrm{D}_{3}$ (cholecalciferol). Vitamin $\mathrm{D}_{3}$ is synthesized in the skin of humans and is consumed in the diet, via the intake of animal-based foods, mainly fish oils. $1,25(\mathrm{OH})_{2}$ vitamin $\mathrm{D}$, the active metabolite of vitamin D (known as calcitriol), regulates not only calcium and phosphate homeostasis, but also cell proliferation and differentiation, and plays a key role in the functioning of the immune and nervous systems. In addition to its classic actions related to mineral homeostasis, vitamin $\mathrm{D}$ also regulates the innate and adaptive immune systems, shows preventive effects on cardiovascular and neurodegenerative diseases and even antiaging effects (Gil et al. 2018).

Aside from having a well-characterized role in calcium and phosphate balance, affecting bone growth and turnover, vitamin D presents other important functions. Thus, its deficiency is associated with increased susceptibility to infectious disease, notably, upper respiratory tract infections (Martineau et al. 2019). In addition, a role for vitamin $D$ in the response to COVID-19 infection could favor the production of antimicrobial peptides in the respiratory epithelium, making infection and the development of COVID-19 symptoms less likely to happen, as well as helping to reduce the inflammatory response to SARS-CoV2 infection. Deregulation of this response, especially of RAS, is characteristic of COVID-19. Vitamin D is known to interact with ACE2, which is also exploited by SARS-CoV-2 as an entry receptor. While SARS-CoV-2 downregulates the expression of ACE2, vitamin D promotes its expression (Mitchel 2020).

Vitamin D deficiency has been associated with an increase in inflammatory cytokines and a significant risk of pneumonia and viral upper respiratory tract infections (Martineau et al. 2019). SARS-CoV-2 promotes a dysfunctional response on the immunological system that dysregulates the cytokine secretory pattern. Hypercytokinemia underlies this hyperinflammatory state leading to injury of alveolar epithelial cells and vascular endothelial cells, as well as to the lung infiltration by neutrophils and macrophages (Pelaia et al. 2020). Thus, the immune system overactivation due to SARS-CoV-2 infection causes the cytokine storm, which is especially noteworthy in severely ill patients (Song et al. 2020; Sun et al. 2020).

Cytokine storm is an acute hyperinflammatory response that may be responsible for critical illness in many conditions including viral infections, cancer, sepsis, and multiorgan failure (Bhaskar et al. 2020). The postmortem examination of lung tissues from COVID-19 patients found proinflammatory cells, involving macrophages and T-helper cells. In addition, evidence shows that SARS-Cov-2 selectively induces a high level of IL-6 resulting in the exhaustion of lymphocytes (Tang et al. 2020).

Vitamin D deficiency is also associated with an increase in thrombotic episodes, frequently observed in COVID-19 (Mohammad et al. 2019). Whether vitamin D does in fact reduce the severity of COVID-19, in regard to pneumonia/ARDS, inflammation, inflammatory cytokines, and thrombosis, it also offers a relatively easy option to decrease the impact of the pandemic (Weir et al. 2020).

Significant relationships have been found between vitamin D levels and the number of COVID-19 cases and especially the mortality caused by this infection (Panagiotou et al. 2020). The most vulnerable group of the population for COVID-19, the aging population, is also the one that has the most deficits of vitamin D levels (Ilie et al. 2020). Additionally, vitamin D has been shown to present anti-inflammatory and antioxidative actions, manifested at peripheral and central sites, as recently demonstrated by us (Almeida Moreira Leal et al. 2020).

Evidence suggests that dopamine and angiotensin II systems counterregulate each other in renal cells, as well as in the striatum and substantia nigra (Labandeira-Garcia et al. 2011). Dopamine depletion may induce RAS upregulation as a potential compensatory mechanism. However, RAS hyperactivation also exacerbates NADPH-oxidase activity, oxidative stress, and the microglial inflammatory response, contributing to dopaminergic neuron loss, as observed with animal models of Parkinson's disease. Manipulation of the brain RAS may constitute an effective neuroprotective strategy against dopaminergic degeneration (Labandeira-Garcia et al. 2011).

In summary, older individuals, especially those with comorbidities, as hypertension and diabetes, have reduced ACE2 expression and upregulation of angiotensin II proinflammatory signaling (Figure 1). It has been hypothesized that with superimposed COVID-19 disease, SARS-CoV-2 binding to ACE2 acutely exaggerates this proinflammatory background, predisposing these subpopulations to greater 


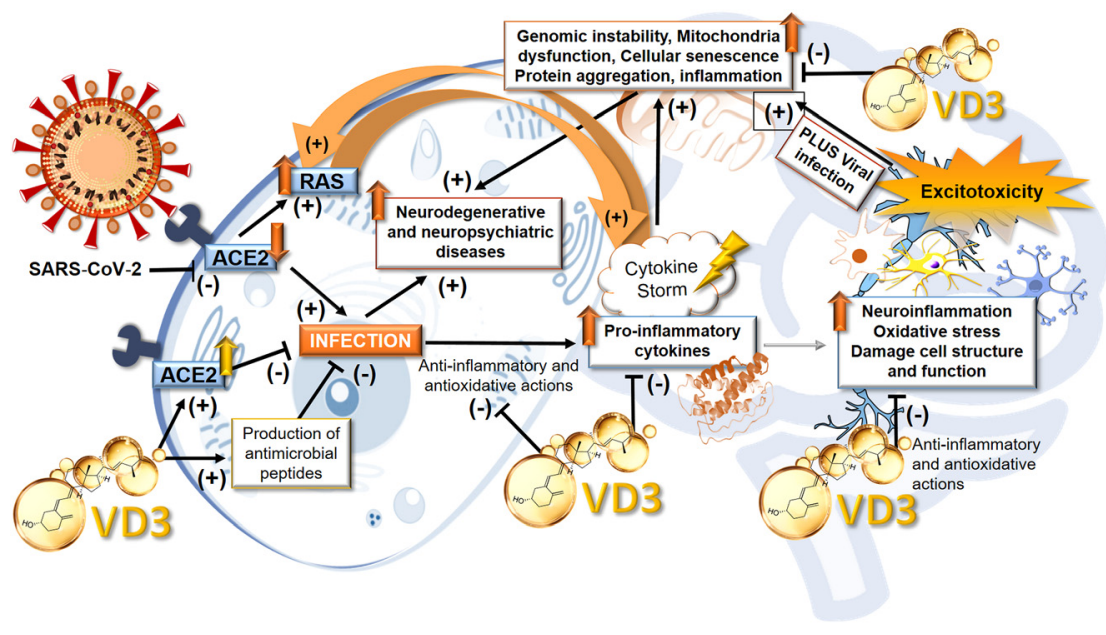

Figure 2: Vitamin D3 supplementation [1,25 $\left.(\mathrm{OH})_{2} \mathrm{D}\right]$ as a possible therapeutic target against COVID-19 neurological symptoms, particularly in individuals with age-related neurodegenerative diseases. Vitamin D3 $\left[1,25(\mathrm{OH})_{2} \mathrm{D}\right]$, the active metabolite of vitamin $\mathrm{D}$, may protect from viral infection and ameliorate the symptoms of COVID-19, preventing the deleterious effects of the cytokine storm. The anti-inflammatory and antioxidative actions of vitamin D3, manifested at peripheral and central nervous sites, help to reduce the inflammatory response to SARS-CoV- 2 infection. Thus, the development of COVID-19-related neurological symptoms is prevented or diminished. Abbreviations: RAS (Reninangiotensin system); ACE2 (angiotensinconverting enzyme 2); VD3 (vitamin D3).

COVID-19 disease severity and mortality (AlGhatrif et al. 2020). There is strong evidence for a major involvement of excessive brain angiotensin-converting enzyme (ACE)/ angiotensin II (Ang II)/angiotensin type-1 receptor (AT-1R) axis, in increased activation of oxidative stress, apoptosis, and neuroinflammation, causing neurodegeneration in several brain disorders. Thus, angiotensin II receptor blockers (ARBs) and activation of ACE2/Angiotensin (1-7)/ MASR axis may serve as an exciting and novel method for neuroprotection, in several neurodegenerative diseases (Obiodun and Ola 2020). The inhibition of ACE by a centrally acting ACE inhibitor has been shown to retard symptoms of neurodegeneration, amyloid-beta plaque formation, and tau hyperphosphorylation, in experimental models of Alzheimer's disease (Quitterer and AbDalla 2019).

Vitamin D, in the form of vitamin D3 (cholecalciferol) supplementation, has potential antiviral properties. Defensins and cathelicidins are the two major families of mammalian antimicrobial proteins that contribute to host, innate, antimicrobial defense by disrupting the integrity of the bacterial cell membrane (Yang et al. 2001). Through several mechanisms, vitamin D can reduce the risk of infections including the induction of cathelicidins and defensins that can lower viral replication rates and reduce the concentrations of proinflammatory cytokines (Grant et al. 2020). Furthermore, vitamin D3 is considered a major factor involved in the regulation of cathelicidin expression and therapies targeting this pathway may provide new treatment modalities in the management of infectious (Schauber and Gallo 2008).

In addition to its immune-system-modulating effects, it has been suggested that vitamin D supplementation plays a role in slowing Parkinson's disease progression and improving the patient's quality of life (Hribar et al. 2020). Although not conducted with neurodegenerative disease patients, there are, to our knowledge, at least two clinical trials already finished: a multicenter randomized and controlled trial of high-dose versus standard-dose vitamin D3 in high-risk COVID-19 patients (Annweiler et al. 2020) and another one (Garzón 2020) focused on the effect of vitamin $\mathrm{D}$ administration on prevention and treatment of mild forms of suspected COVID-19. It has been postulated that conventional oral vitamin D supplementation can be a therapeutic strategy for COVID-19-caused infections by two ways: (i) an attenuation of SARS-CoV-2 infection via downregulation of ACE2 receptor, and (ii) an attenuation of disease severity by decreasing the pulmonary proinflammatory response or cytokine storm that fuels COVID-19 severity (Figure 2). Therefore, its beneficial role assessed by means of epidemiologic, clinical, and experimental evidences may turn vitamin $\mathrm{D}$ a new tool to protect vulnerable populations and mitigate the impact of the current pandemic events (Arbodela and Urcuqui-Inchima 2020). It is worth mentioning that several other trials are at the present moment recruiting subjects to investigate the possible therapeutic role of vitamin $\mathrm{D}$ in different populations (NIH. Studies for vitamin D, COVID19 National Institute of Health NIH 2020).

\section{Conclusions}

This review dealt with studies on COVID-19, focusing on its association with neurodegenerative diseases, such as 
Alzheimer and Parkinson, and the potential therapeutic strategy of vitamin D. SARS-CoV-2 is a neuroinvasive virus responsible for COVID-19 infection and capable of triggering a cytokine storm, which might impact the older population, especially in the presence of neurodegenerative diseases of neuroinflammatory origin (Serrano-Castro et al. 2020). Alzheimer and Parkinson are the two top common neurodegenerative diseases in the elderly (Han et al. 2018) and the COVID-19 pandemic poses a high risk to older people (Naughton et al. 2020). Most importantly, vitamin $\mathrm{D}$ deficiency plays a role in the pathogenesis and course of various neuroinflammatory and neurodegenerative diseases, and there is an association between low levels of vitamin D, Alzheimer, and Parkinson (Koduah et al. 2017). However, there is no consensus on the vitamin D dose needed for treating older people with COVID-19 infection with comorbidities. Evidence indicates that for the treatment of people infected with COVID-19, vitamin D concentrations above $40-60 \mathrm{ng} / \mathrm{mL}$ or $100-150 \mathrm{nmol} / \mathrm{L}$ might be useful (Grant et al. 2020). However, it is important to emphasize that we are dealing with a new clinical condition. The COVID-19-related problems intensify in the agerisk older population, especially in the presence of comorbidities, including neurodegenerative diseases. Thus, randomized controlled trials and large population studies are certainly needed and should be conducted, in order to present more conclusive results.

Acknowledgments: This study was supported by Fundação de Amparo à Pesquisa do Estado de São Paulo (FAPESP) and Conselho Nacional de Desenvolvimento Científico e Tecnológico (CNPq). Bárbara A. Rosário was the recipient of a fellowship grant from Coordenação de Aperfeiçoamento de Pessoal de Nível Superior (CAPES) and Maria de Fátima Santana de Nazaré was the recipient of a fellowship grant from Fundação de Amparo à Pesquisa do Estado de São Paulo (FAPESP).

Author contribution: All the authors have accepted responsibility for the entire content of this submitted manuscript and approved submission.

Research funding: None declared.

Conflict of interest statement: The authors declare no conflicts of interest regarding this article.

\section{References}

Al Ghatrif, M., Cingolani, O., and Lakatta, E. (2020). The dilemma of Coronavirus disease 2019, aging, and cardiovascular disease: insights from cardiovascular aging science. JAMA Cardiol. 5: 747-748.
Almeida Moreira Leal, L.K., Lima, L.A., Alexandre de Aquino, P.E., Costa de Sousa, J.A., Jataí Gadelha, C.V., Felício Calou, I.B., Pereira Lopes, M.J., Viana Lima, F.A., Tavares Neves, K.R., Matos de Andrade, G., et al. (2020). Vitamin D (VD3) antioxidative and anti-inflammatory activities: peripheral and central effects. Eur. J. Pharmacol. 879: 173099.

Annweiler, C., Cao, Z., Wu, Y., Faucon, E., Mouhat, S., Kovacic, H., and Sabatier, J.-M. (2020). Counter-regulatory 'renin-angiotensin' system-based candidate drugs to treat COVID-19 diseases in SARS-CoV-2-infected patients. Infect. Disord. - Drug Targets, https://doi.org/10.2174/1871526520666200518073329 (Epub ahead of print).

Antonini, A., Leta, V., Teo, J., and Chaudhuri, K.R. (2020). Outcome of Parkinson's disease patients affected by COVID-19. Mov. Disord., https://doi.org/10.1002/Fmds.28104 (Epub ahead of print).

Arbodela, J., F., and Urcuqui-Inchima, S. (2020). Vitamin D Supplementation: A Potential Approach for Coronavirus/COVID19 Therapeutics? Front Immunol. 11: 1523.

Baig, A.M. and Sanders, E.C. (2020). Potential neuroinvasive pathways of SARS-CoV-2: deciphering the spectrum of neurological deficit seen in Coronavirus disease-2019 (COVID-19). J. Med. Virol. 92: 1-13.

Bhaskar, S., Sinha, A., Banach, M., Mittoo, S., Weissert, R., Kass, J.S., Rajagopal, S., Pai, A.R., and Kutty, S. (2020). Cytokine Storm in COVID-19 - immunopathological mechanisms, clinical considerations, and therapeutic approaches: the REPROGRAM consortium position paper. Front. Immunol. 11: 1648.

Bhidayasiri, R., Virameteekul, S., Kim, J.-M., Pal, P.K., and Chung, S.-J. (2020). COVID-19: an early review of its global impact and considerations for Parkinson's disease patient care. J. Mov. Disord. 13: 105-114.

Boutoleau-Bretonnière, C., Pouclet-Courtemanche, H., Gillet, A., Bernard, A., Deruet, A.L., Gouraud, I., Mazoue, A., Lamy, E., Rocher, L., Kapogiannis, D., et al. (2020). The effects of confinement on neuropsychiatric symptoms in Alzheimer's disease during the COVID-19 crisis. J. Alzheimer Dis. 76: 41-47.

Cilia, R., Bonvegna, S., Straccia, G., Nico, G., Elia, A., Romito, L., Devigli, G., Cereda, E., and Eleopra, R. (2020). Effects of COVID-19 on Parkinson's disease clinical features: a community-based case-control study. Mov. Disord. 35: 1287-1292.

Daneshkhah, A., Agrawal, V., Eshein, A., Subramanian, H., Roy, H.K., and Backman, V. (2020). The possible role of vitamin D in suppressing cytokine storm and associated mortality in COVID19 patients. MedRxiv, https://doi.org/10.1101/2020.04.08. 20058578.

Davies, G., Garami, A.R., and Byers, J. (2020). Evidence supports a causal role for Vitamin D status in COVID-19 outcomes. MedRxiv, https://doi.org/10.1101/2020.05.01.20087965.

De Felice, F., Tovar-Moll, F., Moll, J., Munoz, D., and Ferreira, S. (2020). Severe acute respiratory syndrome coronavirus 2 (SARS-CoV-2) and the central nervous system. Trends Neurosci. 43: 355-357.

De Chiara, G., Marcocci, M.E., Sgarbanti, R., Civitelli, L., Ripoli, C., Piacentini, R., Garaci, E., Grassi, C., and Palamara, A.T. (2012). Infectious agents and neurodegeneration. Mol. Neurobiol. 46: 614-638.

Ebadi, M. and Montano-Loza, A.J. (2020). Perspective: improving vitamin D status in the management of COVID-19. Eur. J. Clin. Nutr. 74: 856-859. 
Fan, Yi., Zhao, K., Li Shi, Z.-L., and Zhou, P. (2019). Bat coronaviruses in China. Viruses 11: 210.

Fasano, A., Cereda, E., Barichella, M., Cassani, E., Ferri, V., Zecchinelli, A.L., and Pezzoli, G. (2020). COVID-19 in Parkinson's disease patients living in Lombardy, Italy. Mov. Disord. 35: 1089-1093.

Garg, D. and Dhamija, R.K. (2020). The challenge of managing Parkinson's disease patients during the COVID-19 pandemic. Ann. Indian Acad. Neurol. 23: S24-S27.

Grant, W.B., Lahore, H., McDonnell, S.L., Baggerly, C.A., French, C.B., Aliano, J.L., and Bhattoa, H.P. (2020). Evidence that vitamin D supplementation could reduce risk of influenza and COVID-19 Infections and deaths. Nutrients 12: 988.

Ge, X.-Y., Li, J.-L., Yang, X.-L., Chmura, A.A., Zhu, G., Epstein, J.H., Mazet, J.K., Hu, B., Zhang, W., Peng, C., et al. (2013). Isolation and characterization of bat SARS-like coronavirus that uses the ACE2 receptor. Nature 503: 535-538.

Gheblawi, M., Wang, K., Viveiros, A., Nguyen, Q., Zhong, J.-S., Turner, A.J., Raizada, M.-K., Grant, M.B., and Oudit, G.Y. (2020). Angiotensin-converting enzyme 2: SARS-CoV-2 receptor and regulator of the renin-angiotensin system: celebrating the 20th anniversary of the discovery of ACE2. Circ. Res. 126: 1456-1474.

Gil, A., Plaza-Diaz, J., and Mesa, M.D. (2018). Vitamin D: classic and novel actions. Ann. Nutr. Metab. 72: 87-95.

Hainque, E. and Grabli, D. (2020). Rapid worsening in Parkinson's disease may hide COVID-19 infection. Parkinsonism Relat. Disord. 75: 126-127.

Han, Z., Tian, R., Ren, P., Zhou, W., Wang, P., Luo, M., Jin, S., and Jiang, Q. (2018). Parkinson's disease and Alzheimer's disease: a Mendelian randomization study. BMC Med Genet. 19(1): 15.

Harapan, H., Itoh, N., Yufika, A., Winardi, W., Keam, S., Te, H., Megawati, D., Hayati, Z., Wagner, A.L., and Mudatsir, M. (2020). Coronavirus disease 2019 (COVID-19): a literature review. J. Infect. Public Health 13: 667-673.

Herman, C., Mayer, K., and Sarwal, A. (2020). Scoping review of prevalence of neurologic comorbidities in patients hospitalized for COVID-19. Neurology 95: 77-84.

Hofman, H., and Pöhlmann, S. (2004). Cellular entry of the SARS coronavirus. Trends Microbiol. 12(10): 466-472.

Hou, Y., Dan, X., Babbar, M., Wei, Y., Hasselbalch, S.G., Croteau, D.L., and Bohr, V.A. (2019). Ageing as a risk factor for neurodegenerative disease. Nat. Rev. Neurol. 15: 565-581.

Hribar, C.A., Cobbold, P.H., and Church, F.C. (2020). Potential role of vitamin $D$ in the elderly to resist COVID-19 and to slow progression of Parkinson's disease. Brain Sci. 10: 284.

Hu, B., Zeng, L.-P., Yang, X.-L., Ge, X.-Y., Zhang, W., Li, B., Xie, J.-Z., Shen, X.-R., Zhang, Y.-Z., Wang, N., et al. (2017). Discovery of a rich gene pool of bat SARS-related coronaviruses provides new insights into the origin of SARS coronavirus. PLoS Pathog. 13: e1006698.

Ilie, P.C., Stefanescu, S., and Smith, L. (2020). The role of vitamin D in the prevention of coronavirus disease 2019 infection and mortality. Aging Clin. Exp. Res. 32: 1195-1198.

Isaia, G., Marinello, R., Tibaldi, V., Tamone, C., and Bo, M. (2020). Atypical presentation of Covid-19 in an older adult with severe Alzheimer disease. Am. J. Geriatr. Psychiatry 28: 790-791.

Koduah, P., Paul, F., and Dörr, J.-M. (2017). Vitamin D in the prevention, prediction and treatment of neurodegenerative and neuroinflammatory diseases. EPMA Journal 8: 313-325.
Labandeira-Garcia, J.L., Rodriguez-Pallares, J., Villar-Cheda, B., Rodriguez-Perez, A.I., Garrido-Gil, P. and Guerra, M.J. (2011). Aging, angiotensin system and dopaminergic degeneration in the substantia nigra. Aging Dis. 2: 257-274.

Lara, B.B., Carnes, A., Dakterzada, F., Benitez, I., and Piñol-Ripoll, G. (2020). Neuropsychiatric symptoms and quality of life in Spanish patients with Alzheimer's disease during the COVID-19 lockdown. Eur. J. Neurol. 27: 1744-1747.

Lau, E.M.C., Chan, F.W.K., Hui, D.S.C., Wu, A.K.L., and Leung, P.C. (2005). Reduced bone mineral density in male severe acute respiratory syndrome (SARS) patients in Hong Kong. Bone 37: 420-424.

Lau, S.K.P., Luk, H.K.H., Wong, A.C.P., Li, K.S.M., Zhu, L., He, Z., Fung, J., Chan, T.T.Y., Fung, K.S.C., and Woo, P.C.Y. (2020). Possible bat origin of severe acute respiratory syndrome coronavirus 2. Emerg. Infect. Dis. 26: 1542-1547.

Mao, L., Jin, H., Wang, M., Hu, Y., Chen, S., He, Q., Chang, J., Hong, C., Zhou, Y., Wang, D., et al. (2020). Neurologic manifestations of hospitalized patients with coronavirus disease 2019 in Wuhan, China. JAMA Neurol. 77: 683-690.

Martineau, A.R., Jolliffe, D.A., Greenberg, L., Aloia, J.F., Bergman, P., Dubnov-Raz, G., Esposito, S., Ganmaa, D., Ginde, A.A., Goodall, E.C., et al. (2019). Vitamin D supplementation to prevent acute respiratory infections: individual participant data meta-analysis. Health Technol. Assess. 23: 1-44.

Meftahi, G.H., Jangravi, Z., Sahraei, H., and Bahari, Z. (2020). The possible pathophysiology mechanism of cytokine storm in elderly adults with COVID-19 infection: the contribution of “inflame-aging”. Inflamm. Res. 69: 825-839 2020.

Mitchell, F. Vitamin-D and COVID-19: do deficient risk a poorer outcome? Lancet Diabetes Endocrinol. 8: 570, https://doi.org/ 10.1016/s2213-8587(20)30183-2.

Mohammad, S., Mishra, A., and Ashraf, M.Z. (2019). Emerging role of vitamin $D$ and its associated molecules in pathways related to pathogenesis of thrombosis. Biomolecules 9: 649 .

Mok, C.K., Ng, Y.L., Ahidjo, B.A., Lee, R.C.H., Loe, M.W.X., Liu, J., Tan, K.S., Kaur, P., Ching, W.J., and Wong, J.E.-L., et al. (2020). Calcitriol, the active form of vitamin D, is a promising candidate for COVID-19 prophylaxis. BioRxiv Microbiol, https://doi.org/10. 1101/2020.06.21.162396.

Morty, R.E., and Ziebuhr, J. (2020). Call for papers: the pathophysiology of COVID-19 and SARS-CoV-2 infection. Am. J. Physiol. Lung Cell Mol. Physiol. 318: L1016-L1019.

National Institute of Health (NIH) (2020). Studies for vitamin D, COVID19. National Library of Medicine (US), Bethesda (MD).

Naughton, S.H., Raval, U., and Pasinetti, G.M. (2020). Potential novel role of COVID-19 in Alzheimer's disease and preventative mitigation strategies. J. Alzheimer's Dis. 76: 21-25.

Obiodun, O., A., and Ola, M., S. (2020). Role of brain renin angiotensin system in neurodegeneration: An update. Saudi J Biol Sci. 27(3): 905-912.

Ogier, M., Andéol, G., Sagui, E., and Dal Bo, G. (2020). How to detect and track chronic neurologic sequelae of COVID-19? Use of auditory brainstem responses and neuroimaging for long-term patient follow-Up. Brain Behav. Immun. Health 15: 100081.

Panagiotou, G., Tee, S.A., Ihsan, Y., Athar, W., Marchitelli, G., Kelly, D., Boot, C.S., Stock, N., Macfarlane, J., Martineau, A.R., et al. 
(2020). Low serum 25-hydroxyvitamin D (25[OH]D) levels in patients hospitalized with COVID-19 are associated with greater disease severity. Clin. Endocrinol., https://doi.org/10.1111/cen. 14310.(Epub ahead of print).

Pelaia, C., Tinello, C., Vatrella, A., De Sarro, G., and Pelaia, G. (2020). Lung under attack by COVID-19-induced cytokine storm: pathogenic mechanisms and therapeutic implications. Ther. Adv. Respir. Dis. 14: 1-9.

Peña Silva, R.A., Chu, Y., Miller, J.D., Mitchell, I.J., Penninger, J.M., Faraci, F.M., and Heistad, D.D. (2012). Impact of ACE2 deficiency and oxidative stress in cerebrovascular function with aging. Stroke 43: 3358-3363.

Prasad, S., Hola, V.V., Neeraja, K., Surisetti, B.K., Kamble, N., Yadav, R., and Pal, P.K. (2020). Parkinson's Disease and COVID-19: perceptions and implications in patients and caregivers. Mov. Disord. 35: 912-914.

Raony, R., Figueiredo, C.S., Pandolfo, P., Giestal-de-Araujo, E., Bomfim, P.O.-S., and Savino, W. (2020). Psycho-neuroendocrineimmune interactions in COVID-19: potential impacts on mental health. Front. Immunol. 11: 1170

Rejdak, K. and Grieb, P. (2020). Adamantanes might be protective from COVID-19 in patients with neurological diseases: multiple sclerosis, parkinsonism and cognitive impairment. Mult. Scler. Relat. Disord. 42: 102163.

Ruan, Y.J., Wei, C.L., Ee, A.L., Vega, V.B., Thoreau, H., Thoe, S.Y.S., Chia, J.-M., Ng, P., Chiu, K.P., Lim, L., et al. (2003). Comparative full-length genome sequence analysis of 14 SARS coronavirus isolates and common mutations associated with putative origins of infection. Lancet 361: 1779-1785.

Salari, M., Zali, A., Ashrafi, F., Etemadifar, M., Sharma, S., Hajizadeh, N., and Ashourizadeh, H. (2020). Incidence of anxiety in Parkinson's disease during the coronavirus disease (COVID-19) pandemic. Mov. Disord. 35: 1095-1096.

Schauber, J. and Gallo, R.L. (2008). The vitamin D pathway: a new target for control of the skin's immune response? Exp. Dermatol. 17: 633-639.

Serrano-Castro, P.J., Estivill-Torrús, G., Cabezudo-García, P., ReyesBueno, J., Peterson, N.C., Aguillar-Castillo, M.J., Suárez-Pérez, Jimenez-Hernández, M.D., Móya-Molina, M.A., Oliver-Martos, B., et al. (2020). Impact of SARS-CoV-2 infection on neurodegenerative and neuropsychiatric diseases: a delayed pandemic? Neurologia 35: 245-251.

Shalash, A., Roushdy, T., Essam, M., Fathy, M., Dawood, N.L., Abushady, E.M., Elrassas, H., Helmi, A., and Hamid, E. (2020). Mental health, physical activity, and quality of life in Parkinson's disease during COVID-19 pandemic. Mov. Disord. 35: 1097-1099.
Singal, C.M.S., Jaiswal, P., and Seth, P. (2020). SARS-CoV-2, more than a respiratory virus: its potential role in neuropathogenesis. ACS Chem. Neurosci. 11: 1887-1899.

Singhal, T. (2020). A review of coronavirus disease-2019 (COVID-19). Indian J. Pediatr. 87: 281-286.

Sohrabi, C., Alsafi, Z., O’Neil, N., Khan, M., Kerwan, A., Al-Jabir, A., Iosifidis, C., and Agha, R. (2020). World health organization declares global emergency: a review of the 2019 novel coronavirus (COVID-19). Int. J. Surg. 76: 71-76.

Song, P., Li, W., Xie, J., Hou, Y., and You, C. (2020). Cytokine storm induced by SARS-CoV-2. Clin. Chem. Acta 509: 280-287.

Sun, X., Wang, T., Cai, D., Hu, Z., Chen, J., Liao, H., Zhi, L., Wei, H., Zhang, Z., Qiu, Y., et al. (2020). Cytokine storm intervention in the early stages of COVID-19 pneumonia. Cytokine Growth Factor Rev. 53: 38-42.

Tang, Y., Liu, J., Zhang, D., Xu, Z., Ji, J., and Wen, C. (2020). Cytokine storm in COVID-19: the current evidence and treatment strategies. Front. Immunol. 11: 1708.

Vicenzi, E., Canduci, F., Pinna, D., Mancini, N., Carletti, S., Lazzarin, A., Bordignon, C., Poli, G., and Clementi, M. (2004). Corona viridae and SARS-associated coronavirus strain HSR1. Emerg. Infect. Dis. 10: 413-418.

Vieira, J.M., Ricardo, O.M.P., Hannas, C.M., Kanadani, T.C.M., Prata, T.S., and Kanadani, F.N. (2020). What do we know about COVID-19? A review article. Rev. Assoc. Med. Bras. 66: 534-540.

Viel, T., Chinta, S., Rane, A., Chamoli, M., Buck, H., and Andersen, J. (2020). Microdose lithium reduces cellular senescense in human astrocytes - a potencial pharmacotherapy for COVID-19? Aging 12: 10035-10040.

Wang, L., Wang, Y., Ye, D., and Liu, Q. (2020). Review of the 2019 novel coronavirus (SARS-CoV-2) based on current evidence. Int. J. Antimicrob. Agents 55: 105948.

Weir, E.K., Thenappan, T., Bhargava, M., and Chen, Y. (2020). Does vitamin D deficiency increase the severity of COVID-19? Clin. Med. 20: e107-e108.

WHO (2019). Middle East respiratory syndrome coronavirus (MERS(ov). World Health Organization.

WHO (2020). Novel Coronavirus (2019-nCoV): situation report, 19. World Health Organization.

Yang, D., Chertov, O., and Oppenheim, J.J. (2001). Participation of mammalian defensins and cathelicidins in anti-microbial immunity: receptors and activities of human defensins and cathelicidin (LL-37). J. Leukoc. Biol. 69: 691-697.

Zipprich, H.M., Teschner, U., Witte, O.W., Schonenberg, A., and Prell, T. (2020). Knowledge, attitudes, practices, and burden during the COVID-19 pandemic in people with Parkinson's disease in Germany. J. Clin. Med. 9: 16432020. 